\title{
Bacterioplankton assembly and interspecies interaction indicating increasing coastal eutrophication
}

\author{
Wenfang Dai ${ }^{\mathrm{a}, \mathrm{b}}$, Jinjie Zhang ${ }^{\mathrm{a}}$, Qichao Tu ${ }^{\mathrm{c}}$, Ye Deng ${ }^{\mathrm{d}}$, Qiongfen Qiu ${ }^{\mathrm{a}}$, Jinbo Xiong ${ }^{\mathrm{a}, \mathrm{b},{ }^{*}}$ \\ a School of Marine Sciences, Ningbo University, Ningbo, 315211, China \\ b Collaborative Innovation Center for Zhejiang Marine High-Efficiency and Healthy Aquaculture, Ningbo, 315211, China \\ ${ }^{\mathrm{c}}$ Department of Marine Sciences, Ocean College, Zhejiang University, Hangzhou, 310058, China \\ ${ }^{\mathrm{d}}$ Key Laboratory of Environmental Biotechnology, Research Center for Eco-Environmental Sciences, CAS, Beijing, 100085, China
}

\section{H I G H L I G H T S}

- Increasing coastal eutrophication linearly increases the importance of deterministic processes that constrained BCCs.

- The buffer capacity of BCCs is limited, with disrupted interspecies interaction occurring under heavy eutrophication.

- The dynamics of bio-indicators closely linked with eutrophication levels and are concordant with their ecological functions.

\section{A R T I C L E I N F O}

\section{Article history:}

Received 9 November 2016

Received in revised form

26 February 2017

Accepted 9 March 2017

Available online 9 March 2017

Handling Editor: Caroline Gaus

\section{Keywords:}

Coastal eutrophication

BCCs

Spatial distribution

Deterministic processes

Interspecies interaction

Bio-indicators

\begin{abstract}
A B S T R A C T
Anthropogenic perturbations impose negative effects on coastal ecosystems, such as increasing levels of eutrophication. Given the biogeochemical significance of microorganisms, understanding the processes and mechanisms underlying their spatial distribution under changing environmental conditions is critical. To address this question, we examined how coastal bacterioplankton communities respond to increasing eutrophication levels created by anthropogenic perturbations. The results showed that the magnitude of changes in the bacterioplankton community compositions (BCCs) and the importance of deterministic processes that constrained bacterial assembly were closely associated with eutrophication levels. Moreover, increasing eutrophication significantly $(P<0.001)$ attenuated the distance decay rate, with a random spatial distribution of BCCs in the undisturbed location. In contrast, the complexity of interspecies interaction was enhanced under moderate eutrophication levels but declined under heavy eutrophication. Changes in the relative abundances of 27 bacterial families were significantly correlated with eutrophication levels. Notably, the pattern of enrichment or decrease for a given bacterial family was consistent with its known ecological functions. Our findings demonstrate that the magnitude of changes in BCCs and underlying determinism are dependent on eutrophication levels. However, the buffer capacity of bacterioplankton community is limited, with disrupted interspecies interaction occurring under heavy eutrophication. As such, bacterial assemblages are sensitive to changes in environmental conditions and could thus potentially serve as bio-indicators for increasing eutrophication.
\end{abstract}

(c) 2017 Elsevier Ltd. All rights reserved.

\section{Introduction}

Patterns of anthropogenic coastal use have undergone considerable changes in recent times, with the advent and expansion of coastal aquaculture, urban, agricultural, and industrial runoff (Fodelianakis et al., 2014; Xiong et al., 2015). As a result, coastal

\footnotetext{
* Corresponding author. School of Marine Sciences, Ningbo University, Ningbo, 315211, China.

E-mail address: xiongjinbo@nbu.edu.cn (J. Xiong).
}

ecosystems are being threatened by increasing eutrophication (SOA, 2015). Currently, multivariate index has been widely applied to classify water quality. (Primpas et al., 2010; Hou et al., 2016). However, this index does not take into consideration additive, synergistic and antagonistic effects of biota and abiotic factors (Borruso et al., 2015). Microbial communities are generally the first responders to environmental perturbation and can either augment or buffer environmental changes (Labbate et al., 2016). Thus, the application of bacterial bio-indicator could systematically reflect and record both biotic and abiotic (including unmeasured and 
unobserved factors) pressures, even after the contaminants themselves have been fully degraded (Smith et al., 2015). Indeed, natural bacterial communities have been widely applied as in situ environmental indicators that respond to and capture anthropogenic perturbations (Fodelianakis et al., 2014; Xiong et al., 2014; Borruso et al., 2015). Nevertheless, no consensus has been emerged regarding the microbial resistance and resilience to a changing environment (Allison and Martiny, 2008; Shade et al., 2012). For example, consistent associations of specific bacterioplankton groups with nutrient enrichment are detected across different habitats (Fodelianakis et al., 2014), and seasons (Xiong et al., 2015). In contrast, it was reported that microbial community resists perturbation by nutrient enrichment (Bowen et al., 2011). This apparent discrepancy could be due to the sensitivity of microbial communities depending on the eutrophication level (Van Der Gast et al., 2008; Shade et al., 2012). Consistently, both field surveys and laboratory experiments have shown that the magnitude of changes in microbial communities is dependent on pollution levels (Ager et al., 2010; Xiong et al., 2014). However, it is unknown whether this pattern would be disrupted under severe disturbance despite such information being crucial for evaluating the buffer capacity of an ecosystem to increasing anthropogenic perturbations. Recently, it has been proposed that the response of microbial communities to environmental changes is structured by a complex combination of adaptation (e.g., resistance and resilience), replacement (e.g., immigrants dispersed from nearby localities), and interspecies interaction mechanisms (Shade et al., 2012; Xiong et al., 2015; Deng et al., 2016). For these reasons, a better understanding of the ecological processes affecting microbial communities is vital for predicting the responses of ecosystems to a changing environment.

Recently, the distance-decay relationship (DDR) has been applied to quantify the relative robustness of community response to organic pollution (Liang et al., 2015). The slope of the linear regression between logarithmic $\beta$-similarities and logarithmic geographic distances reflects the distance decay rate (Nekola and White, 1999), which provides a descriptive statistic for the change rate and therefore can be used to track the stability of a microbial community (Shade et al., 2012). For example, long-term oil exposure significantly retards the distance decay rate of the microbial functional composition (Liang et al., 2015). Two types of processes (deterministic vs. stochastic) have been proposed to explain the DDR (Nemergut et al., 2013; Zhou et al., 2014). Specifically, the spatial environmental heterogeneity selects and sorts microorganisms by deterministic fitness differences between taxa (Van der Gucht et al., 2007); however, this effect could be overridden by stochastic spatial dispersal (e.g., high dispersal rates at local scales due to hydraulic conductivity) of planktonic microorganisms (Finlay, 2002). It is now recognized that deterministic and stochastic processes jointly shape microbial assembly, although their relative importance is still being actively debated (Stegen et al., 2012; Xiong et al., 2015). For example, it has been proposed that nutrient input increases bacterial compositional stochasticity by enhancing ecological drift and weakening species sorting (Zhou et al., 2014), whereas, in contrast, studies show that the increasing concentrations of industrial wastewater contribute a gradual shift from stochastic to more deterministic-based processes (Van Der Gast et al., 2008). These divergent conclusions raise the question of whether and how the relative importance of deterministic processes was affected by increasing coastal eutrophication.

In a given habitat, diverse microbial assemblages interact with one another to form complicated networks through various types of interactions, such as competition and/or mutualism (Faust and Raes, 2012). As such, interspecies interaction determines the responses of a microbial community to disturbance (Shade et al.,
2012; Dang and Lovell, 2016). While deterministic processes (e.g., disturbance-induced environmental filtering) select taxa that are more closely related to co-occurring relatives than would be expected by chance, thereby revealing niche spaces shared by community members (Stegen et al., 2012; Monier et al., 2015). The strength of links among species changes along environmental gradients such that it is increasingly important to determine the buffer capacity to heavy eutrophication. In addition, it has been reported, albeit limited, that synergistic interspecies interaction is critical in maintaining proper functions (Zhu et al., 2016). The complexity of interspecies interaction (e.g., proportion of positive co-occurrence, network size, average clustering coefficient) may therefore provide unique indices for evaluating the stability of microbial communities (Deng et al., 2012; Shade et al., 2012). Given that interaction analyses in microbial ecology are still in its infancy, it is unclear to what extent that the complexity of interspecies interaction is altered with increasing eutrophication.

Coastal areas of the East China Sea are strongly affected by anthropogenic activities, resulting in a complex mixture of pollutants (SOA, 2015). There is ample evidence that the bacterioplankton community compositions (BCCs) are markedly affected by both organic and inorganic pollutants (Xiong et al., 2015; Pringault et al., 2016). However, using a forward selection, our previous work showed that the BCCs were primarily determined by the nutrient variables, rather than the measured heavy metals (Wang et al., 2015). For this reason, here we only explored how eutrophication affects the assembly of bacterial community. To achieve this, we integrated the DDR model (Nekola and White, 1999), the standardized effect size measure (Stegen et al., 2013), and network inference approaches (Deng et al., 2012) to address the following questions: (i) how eutrophication affect the spatial patterns of bacterioplankton communities? (ii) to what extent is the relative importance of deterministic processes altered by increasing eutrophication? and (iii) what is the relationship between the strength of interspecies interaction and eutrophication levels? In addition, given that a bacterial bio-indicator provides quantitative information on the eutrophication level around it (Borruso et al., 2015), we further screened sensitive taxa for indicating eutrophication level. To this end, Illumina sequencing data for a spatial survey of bacterioplankton communities were collected from our previous work, in which showed that the biogeography of bacterial community was determined by spatially structured environmental gradients (Wang et al., 2015). In contrast, here we explored to what extent that the assembly (e.g., determinism and interspecies interaction) of bacterial community was affected by increasing coastal eutrophication from an ecological perspective. This further work revealed that increasing coastal eutrophication linearly enhanced the importance of deterministic processes that constrained BCCs. However, the buffer capacity of bacterioplankton community was disrupted under heavy eutrophication. In addition, bio-indictors were identified to indicate eutrophication levels.

\section{Materials and methods}

\subsection{Data collection and location descriptions}

The sequencing data were obtained from the Sequence Read Archive of DDBJ (http://www.ddbj.nig.ac.jp), under the open accession number DRA002865. Briefly, the V4 region of bacterial 16S rDNA gene were amplified and paired-end sequenced on an Illumina MiSeq machine (Wang et al., 2015). Biogeochemical data were downloaded from the supplemental information (Wang et al., 2015). We selected four representative locations consisting of Yushan Reserve, Sanmen Bay, Xiangshan Bay and Hangzhou Bay (Fig. S1). All samples were collected within two weeks in summer 
to negate the effects of seasonality on BCCs.

Yushan Reserve is a marine ecological special protection zone, where disturbances by anthropogenic activities are prohibited. The nutrient level in this zone is very low (Table S1, Wang et al., 2015), thus we designated it as the control location.

Sanmen Bay and Xiangshan Bay are semi-enclosed bays connected to the East China Sea; the lands surrounding the two bays have been farmed for decades. In addition, two coal-fired power plants are situated at the end (Guohua power plant) and middle (Wusha Mountain power plant) of Xiangshan Bay, which began operations in December 2005 and 2006, respectively.

Hangzhou Bay is located downstream of the Qiantang River and south of the Yangtze River estuary and is thus subjected to enormous volumes of water and sediment discharges from the two rivers annually. The runoffs were estimated be $4.25 \times 10^{4} \mathrm{t}$ nitrate and $0.67 \times 10^{3}$ t phosphate per year, respectively (SOA, 2015).

\subsection{Eutrophication index estimation}

The eutrophication index (EI) was calculated as: $\mathrm{EI}=\mathrm{DIN} \times \mathrm{DIP} \times \mathrm{COD} \times 10^{6} / 4500($ AQSIQ 2007$)$, where DIN is the dissolved inorganic nitrogen content (the sum level of ammonia, nitrate and nitrite, expressed in $\mathrm{mg} / \mathrm{L}$ ), DIP is the dissolved inorganic phosphorus content (mg/L), and COD is the chemical oxygen demand $(\mathrm{mg} / \mathrm{L})$. The geochemical variables for EI calculation were listed in Table S1. Eutrophication levels were categorized as follows: mild eutrophication ranged from $1.0 \leq \mathrm{EI} \leq 3.0$; moderate eutrophication ranged from $3.0<\mathrm{EI} \leq 9.0$; and heavy eutrophication ranged from $\mathrm{EI}>9.0$ (The specification for marine monitoring-Part 4: Seawater analysis) (AQSIQ 2007). This index integrates the indirect ecological effects of COD concentration, thus it is better than the traditionally-used water quality index that is primarily based on the essential nutrients.

\subsection{Statistical analysis}

To correct for uneven sequencing efforts and to avoid biases associated with repeatedly subsampling the smallest library, we used a randomly selected subset of 11,400 sequences (corresponding to $80 \%$ of the smallest library size) per sample to calculate distances between samples. Non-metric multidimensional scaling (NMDS) and analysis of similarity (ANOSIM) based on UniFrac distances were performed to evaluate the overall differences in the BCCs (Kenkel and Orlóci, 1986). To screen sensitive bacterial assemblages that were significantly associated with eutrophication levels, Pearson correlation coefficients between the relative abundances of bacterial family and EI were calculated using SPSS 13.0 (SPSS Inc, Chicago, IL, USA). The significant coefficients $(P<0.05)$ were visualized in a heat map using the pheatmap package in $\mathrm{R}$ project (Kolde, 2012; R Development Core Team, 2014). To quantify the phylogenetic diversities (i.e., phylobetadiversity), the mean nearest taxon distance (MNTD) were calculated across the sample (Stegen et al., 2013). MNTD is the mean phylogenetic distance separating each species in the community from its closest relative, which is sensitive to changes in lineage close to the phylogenetic tips (Kembel et al., 2010). To evaluate the degree of non-random phylogenetic relatedness, the weighted standardized effect size of the phylogenetic community structure (ses.MNTD) was calculated for MNTD based on the difference between phylogenetic distances in the observed communities versus null communities generated with 999 randomizations divided by the standard deviation of phylogenetic distances in the distribution (Webb et al., 2002; Kembel et al., 2010). Negative ses.MNTD values with low quantiles $(P<0.05)$ indicate that a community is more closely related than expected by chance (determinism), whereas positive values with high quantiles $(P>0.95)$ indicate that a community is less closely related than expected by chance (stochasticity) (Webb et al., 2002; Stegen et al., 2013). A larger absolute magnitude of ses.MNTD value reflects the stronger effects of deterministic processes (Stegen et al., 2013; Zhu et al., 2016). These analyses were implemented in the R environment with the package 'Picante' (Kembel et al., 2010; R Development Core Team, 2014). To compare the effects of increasing eutrophication on bacterial community assembly, we evaluated the distance decay rate and interspecies interaction for each location separately. The slope (distance decay rate) was estimated with a linear regression between the geographic distance among samples and similarity in BCCs (Horner-Devine et al., 2004; Xiong et al., 2014). To test if the slope of DDR was different from zero, bootstrapping was used for regressing variables that violate the assumption of independence. A mean of bootstrapped slopes by random pairing of the original set (999 times with replacement) for each location was calculated (HornerDevine et al., 2004), the significance of distance decay rate among locations was tested by a one-way ANOVA (analysis of variance). A random matrix theory-based approach was used to evaluate the effects of increasing eutrophication on the bacterial interspecies interactions, using an open-access pipeline available at http://ieg2. ou.edu/MENA (Deng et al., 2012). Hub and connector taxa were determined by among-module connectivity $\left(P_{i}\right)$ and within-module connectivity (Shannon et al., 2003), with nodes categorized into module hubs $\left(P_{i} \leq 0.62\right.$ and $\left.Z_{i}>2.5\right)$, connectors $\left(P_{i}>0.62\right.$ and $\left.Z_{i} \leq 2.5\right)$, network hubs $\left(P_{i}>0.62\right.$ and $\left.Z_{i}>2.5\right)$ and peripheral nodes $\left(P_{i} \leq 0.62\right.$ and $\left.Z_{i} \leq 2.5\right)$, as proposed previously (Olesen et al., 2006; Deng et al., 2012).

\section{Results}

\subsection{Eutrophication levels among locations}

Eutrophication levels were markedly different among the four locations, with the lowest EI recorded at Yushan Reserve (ranged from 0.03 to $1.07,0.43 \pm 0.15$, mean \pm standard deviation), and the highest at Hangzhou Bay (ranged from 20.97 to 51.65, $33.53 \pm 9.10$ ) (Table S1 and Fig. 1), indicating the effectiveness of specialprotection status in maintaining the ecological systems of marine

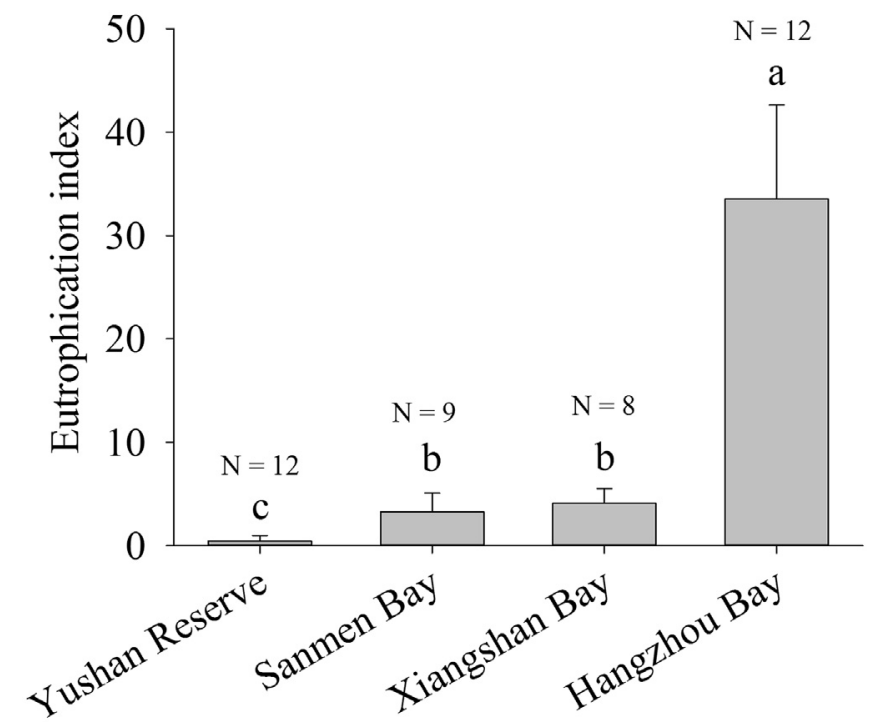

Fig. 1. The mean ( \pm standard deviation) of the eutrophication indices of the four sampling locations. The sampling size for each location was shown. Different small letters indicate significant differences between locations. 
environments. In addition, the EI values were comparable between Sanmen Bay (ranged from 0.85 to $11.82,4.09 \pm 1.44$ ) and Xiangshan Bay (ranged from 2.91 to $7.32,3.25 \pm 1.84$ ) (Table S1 and Fig. 1), which were congruent with their similar intensities of anthropogenic activity, such as intensive aquaculture.

\subsection{Spatial distribution of bacterioplankton communities}

Based on the operational taxonomic units (OTUs) detected across the samples, an NMDS ordination analysis clearly revealed the differentiation in BCCs among locations (Fig. 2A). This pattern was further supported by the results of an analysis of similarity (ANOSIM, Global $r=0.932, P<0.001$ ), which demonstrated that the BCCs were significantly distinct between each pair of locations being compared (Table S2). Notably, the dissimilarities among the bacterial communities were significantly correlated with eutrophication levels (Fig. 2B), suggesting that the magnitude of changes in BCCs is dependent on the selective pressures exerted by increasing eutrophication. In addition, positive correlations were detected between bacterial $\alpha$-diversity and EI (Table S3).

No significant correlation was detected between geographic distance and community similarities for the Yushan Reserve samples (Fig. 3A). The slope is not significantly different from 0 at this control location. In contrast, the spatial distribution of
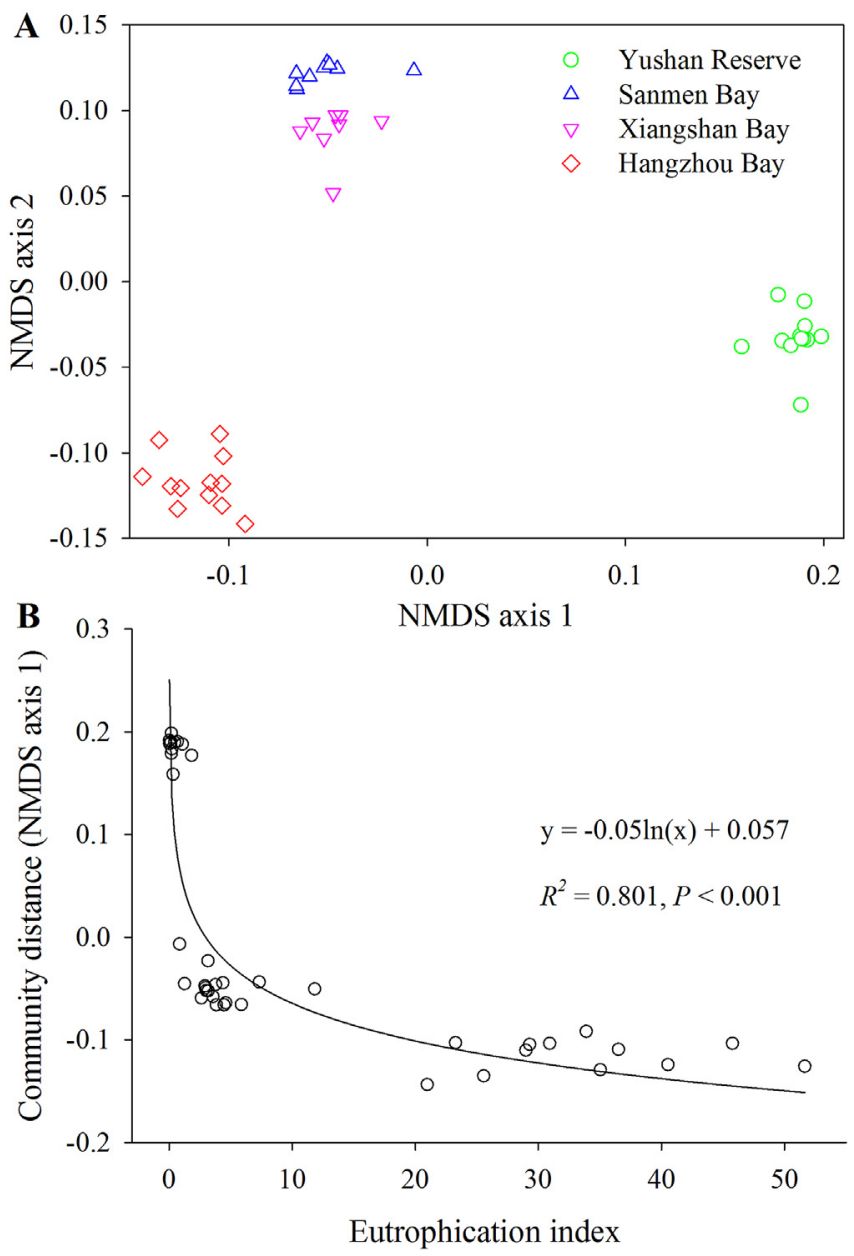

Fig. 2. Non-metric multidimensional scaling (NMDS) plot derived from UniFrac distances between samples, with symbols coded by sampling location (A), and a logarithmic regression fit the first component from NMDS (a proxy of community distances) and eutrophication index community (B). bacterioplankton communities in the disturbed locations followed the DDR pattern $(P<0.05$ in all cases) (Fig. 3). In addition, the distance decay rate of the bacterioplankton communities in Hangzhou Bay samples was significantly lower $(P<0.001)$ than those in samples from Xiangshan Bay and Sanmen Bay.

\subsection{Elevated determinacy along increasing eutrophication}

In order to evaluate how increasing eutrophication affects the relative importance of deterministic processes in shaping BCCs, ses.MNTD was calculated for each sample. The ses.MNTD values of BCCs in Yushan Reserve were around zero, suggesting that the assembly of bacterial communities is stochastic in control location. In contrast, BCCs from the disturbed locations were mainly governed by local environmental filtering, as most ses.MNTD values for the communities were less than -2 . In addition, the deterministic processes that shaped the community assembly tended to strengthen significantly (regression models, $r=-0.838, P<0.001$ ) with increasing coastal eutrophication (Fig. 4).

\subsection{Identification of sensitive bacterial families for characterizing eutrophication levels}

Given that BCCs were dramatically altered by increasing eutrophication, we then asked whether bacterial assemblages could be indicative for eutrophication levels. As such, we screened 27 bacterial families for which their relative abundances were significantly associated with EI (Fig. 5). However, the responses of these assemblages to increasing eutrophication were divergent; for example, the relative abundances of bacterial families affiliated with Bacteroidetes (including the Cryomorphaceae, Flavobacteriaceae and Saprospiraceae) were negatively correlated with eutrophication levels, whereas assemblages belonging to Betaproteobacteria (Comamonadaceae and Methylophilaceae) were positively correlated with eutrophication levels (Fig. 5). Notably, there were clear distinctions between the samples (with the exception of sample S118) based on the occurrence of these 27 bacterial families (Fig. 5). Thus, these sensitive assemblages have the potential to be used as bio-indicators of eutrophication levels.

\subsection{Networks revealing hubs of interspecies interaction}

To ensure correlation reliability, the bacterial OTUs were reduced by retaining only those detected in more than $50 \%$ of the samples within each location, resulting in 330, 470, 726 and 668 OTUs for Yushan Reserve, Sanmen Bay, Xiangshan Bay and Hangzhou Bay, respectively (Table 1). The network connectivity distribution curves had comparable similarity thresholds when plotted and fitted with the power-law model ( $\mathrm{R}^{2}$ range of $0.788-0.956$ ) (Table 1), suggesting that the constructed networks were scale-free. The average clustering coefficient (avgCC), average degree (avgK), and positive co-occurrence of Sanmen Bay and Xiangshan Bay were higher than those in Yushan Reserve (Table 1). In contrast, the topological properties were comparable between Hangzhou Bay and Yushan Reserve (Table 1). Notably, the topological roles of bacterial taxa were distinct among locations; for instance, there were more module hubs in Hangzhou Bay than in other locations (Fig. 6). Taken together, moderate eutrophication levels (Sanmen Bay and Xiangshan Bay) appear to enhance interspecies interaction, whereas heavy eutrophication could disrupt such interactions.

\section{Discussion}

The increasing incidence and severity of coastal eutrophication is the trajectory to be expected. Given the functional importance of 

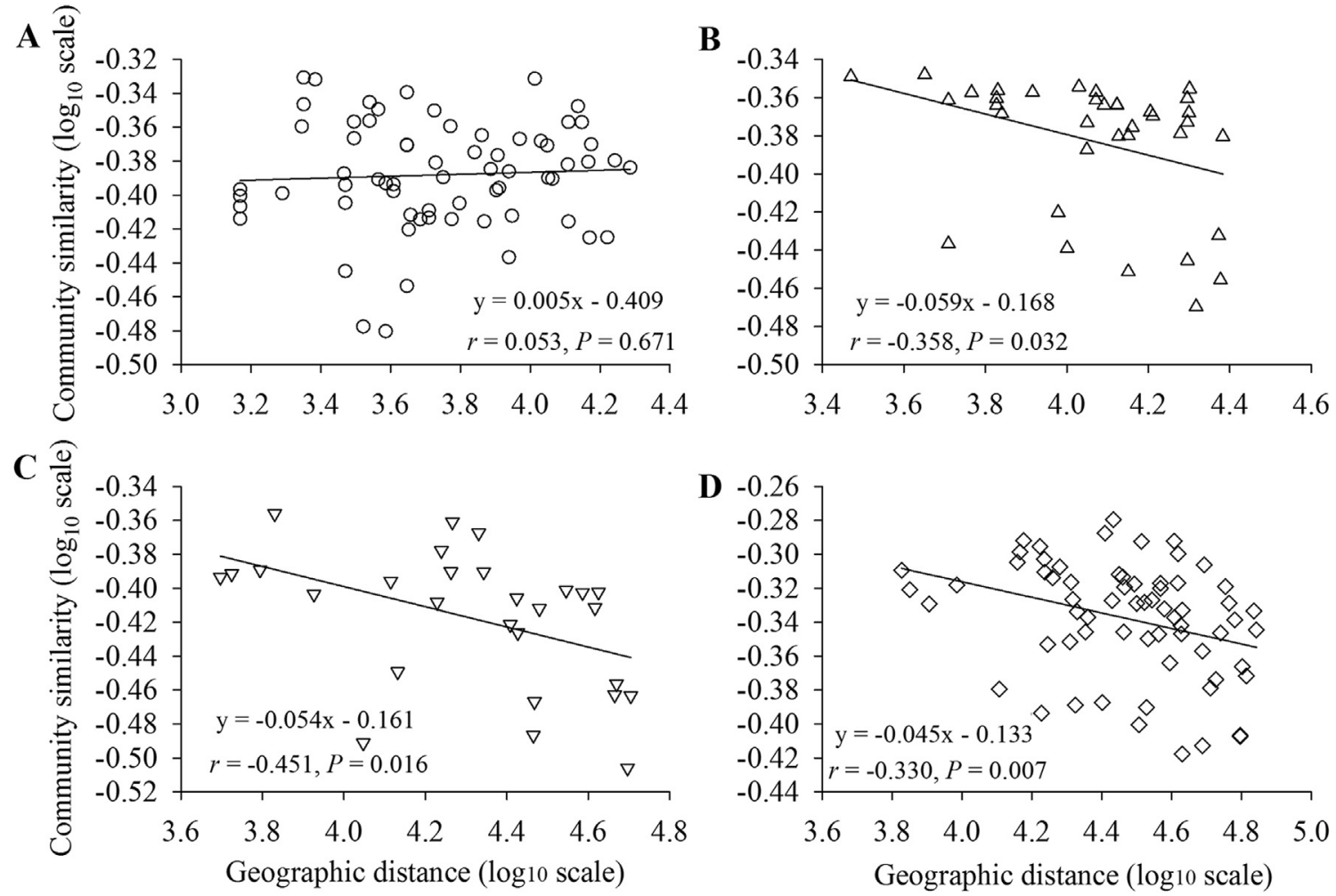

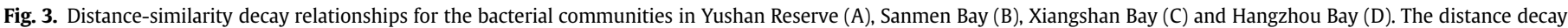
rate, $w$ (the regression slope), was estimated directly using a linear regression (log-log space approach) fit between pairwise similarity and geographic distance.

microorganisms in biogeochemical cycling, understanding how the assembly of bacterial community in response to increasing coastal eutrophication is clearly of great interest. Bacterioplankton communities have long been known to have a high degree of physiological plasticity (Allison and Martiny, 2008; Shade et al., 2011), thus resulting in no or bell-shaped-curve disturbance-community relationships (Hall et al., 2012). However, we found that increasing eutrophication levels drove gradually stronger changes in BCCs (Fig. 2), a pattern that has also been detected with changes along

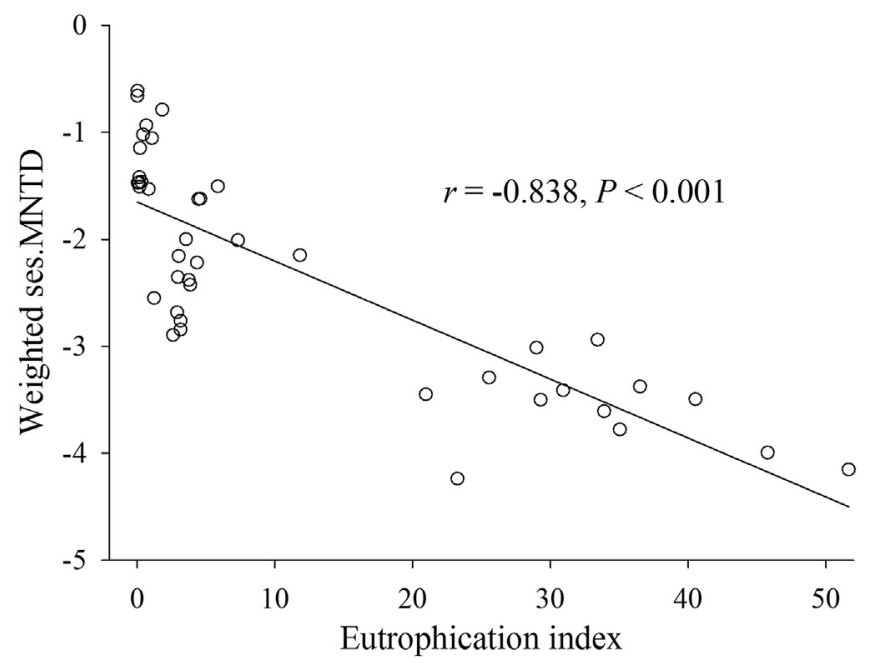

Fig. 4. The correlation between the importance of deterministic processes (as measured by the weighted standardized effect size of the mean nearest taxon distance, ses.MNTD) of the bacterial communities and increasing eutrophication index. salinity, temperature and pollution gradients (Ager et al., 2010; Berga et al., 2012; Xiong et al., 2016). Increasing evidence has shown that long-term coastal nutrient pollution markedly alters the composition (Fodelianakis et al., 2014), assembly (Xiong et al., 2015) and function (Zhang et al., 2015) of bacterioplankton community. Thus, the long-term coastal eutrophication in our study locations (disturbed by anthropogenic activities for decades and centuries) could be characterized as "press" events (Shade et al., 2012). The press disturbance could select species with fitting biological traits and exclude (filters out) other members from the endemic species pool (Lepori and Malmqvist, 2009; Stegen et al., 2012). Consistent with this notion, fast-growing taxa (copiotrophs) would have a competitive advantage than that of oligotrophs. Consistently, a high relative abundance of copiotrophic $\beta$ Proteobacteria was detected in the heavy eutrophication Hangzhou Bay (Wang et al., 2015). It is widely accepted that the level of microbial community resistance is dependent on the harshness of disturbance (Chase, 2007; Lepori and Malmqvist, 2009). Under these premises, the positive relationship between changes in BCCs and eutrophication levels detected here (Fig. 2B) thus provides a basis for predicting the responses of bacterioplankton communities to a changing environment.

Coastal eutrophication contributed a DDR pattern as opposed to a random spatial distribution of the bacterial community in the control location (Fig. 3). There are several possible reasons why we observed such an apparent difference. First, one might argue that the spatial pattern depends on the spatial scale (Martiny et al., 2011), while this is not the case here, as the scale of geographic distances within each location is similar (Fig. 3). Another possible explanation for the random distribution, then, is that stochastic colonization exerted a dominated role in shaping BCCs, leading to the homogenization of bacterial taxa in Yushan Reserve (Fig. 3). 


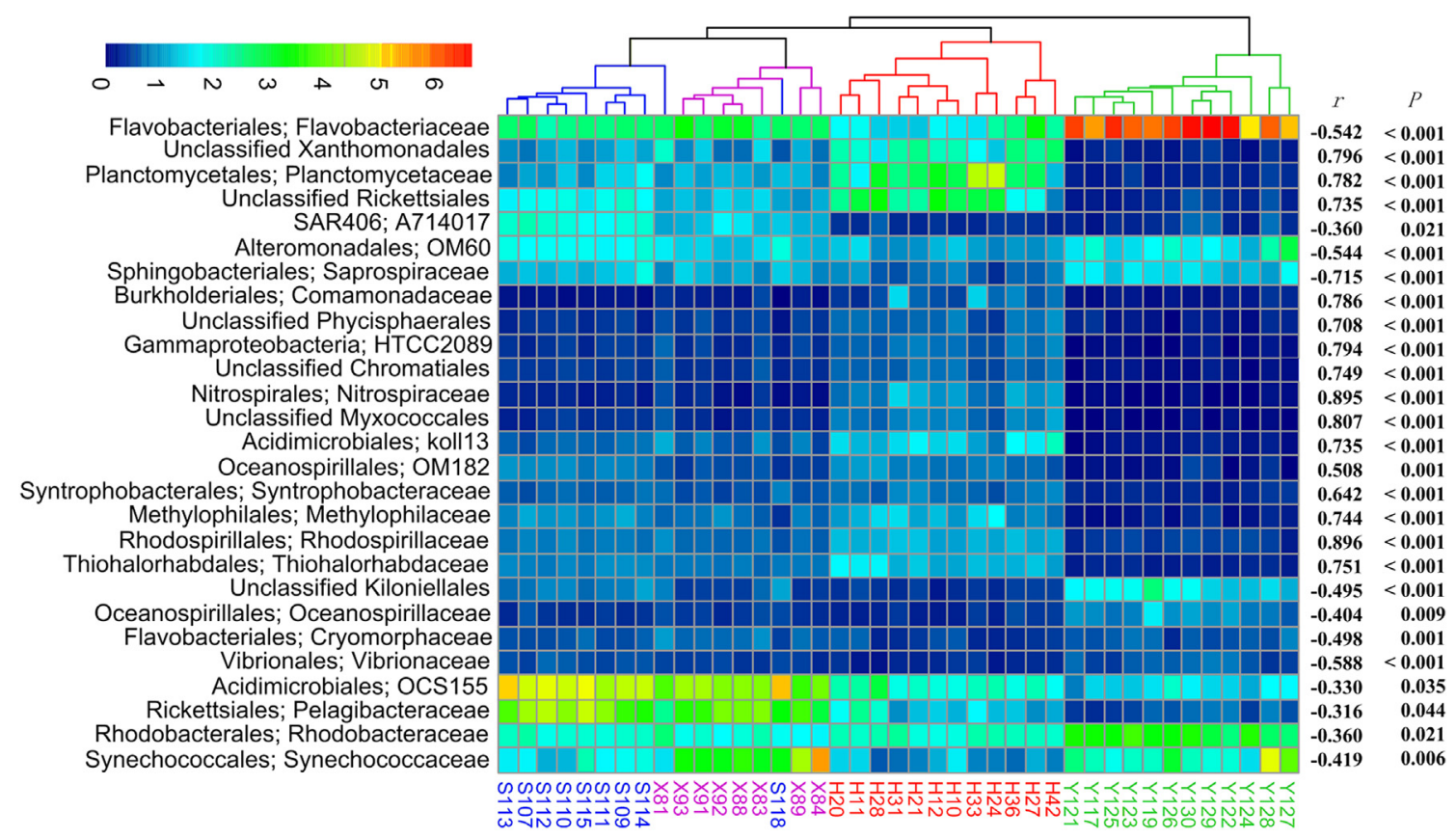

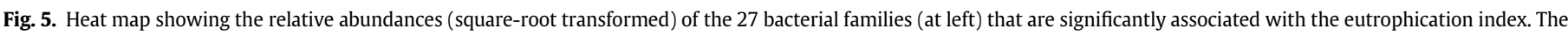

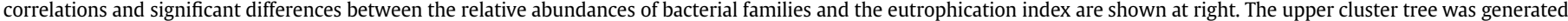

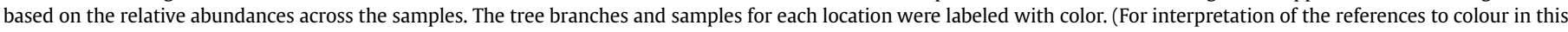
figure legend, the reader is referred to the web version of this article.)

Third, the rapid dispersal ability (mass effect) of microorganisms contributes a random distribution of microbial community (Finlay, 2002). By contrast, increasing eutrophication pressures select (i.e., niche selection) tolerant species from its endemic pool, resulting in the legacy effects associated with geographic distance at the disturbed locations (Fig. 3). This assertion is supported by our previous finding that environmental variables exhibited clear spatially structured patterns (Wang et al., 2015). In addition, higher levels of eutrophication drive the BCCs from oligotroph-dominated to copiotroph-dominated assemblages, thereby resulting in marked changes in BCCs (Fig. 2). It is interesting to note that the distance decay rates were attenuated by increasing eutrophication levels (Fig. 3). Thus, it is likely that moderate eutrophication induces the selectivity and stability of bacterioplankton communities, thereby resulting in a lower $\beta$-diversity (more convergent communities) (Fig. 2). To our knowledge, no study has established the relationship between spatial pattern of BCCs and increasing eutrophication levels in field, although diminished rates of temporal turnover across pollution gradients have previously been detected in microcosms (Van Der Gast et al., 2008; Ager et al., 2010).

It is now generally accepted that deterministic and stochastic processes jointly control the $\beta$-diversity of local communities (Liang et al., 2015; Xiong et al., 2015), but uncertainty remains about their relative importances along disturbance intensity (Lepori and Malmqvist, 2009; Stegen et al., 2012). For example, it has been shown that deterministic processes guide the long-term synchronized dynamics of bacterial community in anaerobic digesters (Vanwonterghem et al., 2014); whereas a short-term study finds that stochastic assembly plays dominant roles in creating the variations of BCCs and functions (Zhou et al., 2013). This divergence is consistent with the notion that bacterioplankton assemblages are resilient to short-term pulse disturbance (Shade et al., 2011). In addition, it has been reported that bacterioplankton communities are stochastic in the absence of drought, while are deterministically structured in ponds that experienced drought (Chase, 2007). Accordingly, the importance of deterministic environmental

Table 1

Topological properties of the empirical molecular ecological networks (MENs) of bacterial communities and their associated random MENs.

\begin{tabular}{|c|c|c|c|c|c|}
\hline & \multirow[t]{2}{*}{ Topological properties } & \multicolumn{4}{|l|}{ Location } \\
\hline & & Yushan Reserve & Sanmen Bay & Xiangshan Bay & Hangzhou Bay \\
\hline \multirow[t]{10}{*}{ Empirical networks } & Similarity threshold (st) & 0.820 & 0.870 & 0.910 & 0.880 \\
\hline & Network size $(\mathrm{n})$ & 330 & 470 & 726 & 668 \\
\hline & Links $(\mathrm{n})$ & 494 & 1596 & 2196 & 758 \\
\hline & Links per node & 1.5 & 3.4 & 3.0 & 1.1 \\
\hline & $\mathrm{R}^{2}$ of power law & 0.942 & 0.788 & 0.844 & 0.956 \\
\hline & Average path (GD) & 3.896 & 3.161 & 5.992 & 3.638 \\
\hline & Average clustering coefficient (avgCC) & 0.089 & 0.170 & 0.145 & 0.071 \\
\hline & Average degree (avgK) & 2.994 & 6.791 & 6.050 & 2.269 \\
\hline & Positive co-occurrence & $39.1 \%$ & $56.9 \%$ & $66.0 \%$ & $44.8 \%$ \\
\hline & Modularity (M) & 0.748 & 0.817 & 0.863 & 0.854 \\
\hline \multirow[t]{3}{*}{ Random networks } & Average path (GD) & $4.073 \pm 0.190$ & $3.022 \pm 0.084$ & $3.617 \pm 0.050$ & $3.447 \pm 0.228$ \\
\hline & Average clustering coefficient (avgCC) & $0.012 \pm 0.005$ & $0.127 \pm 0.009$ & $0.018 \pm 0.003$ & $0.006 \pm 0.002$ \\
\hline & Modularity (M) & $0.616 \pm 0.008$ & $0.301 \pm 0.004$ & $0.379 \pm 0.004$ & $0.761 \pm 0.006$ \\
\hline
\end{tabular}




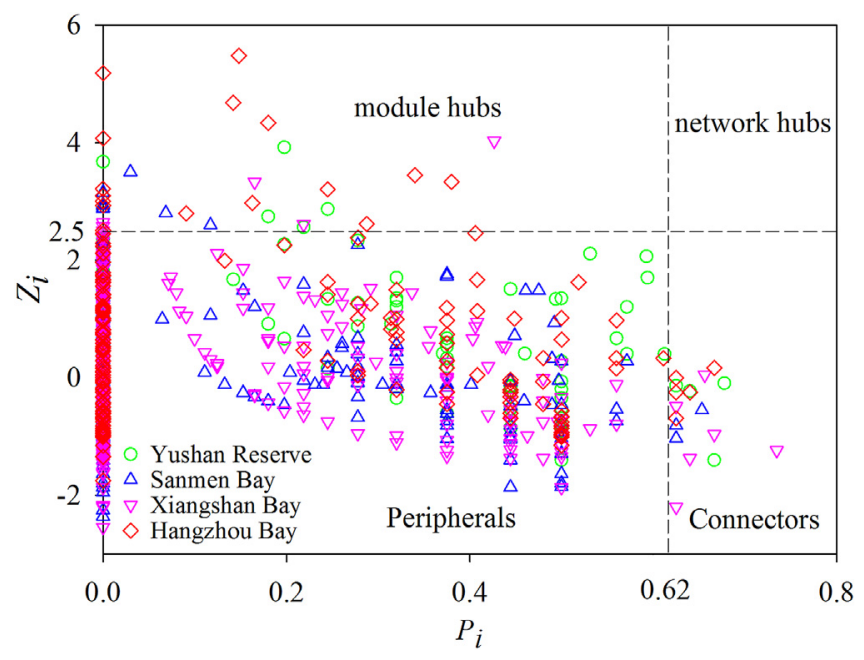

Fig. 6. Z-P plot showing the distribution of bacterial taxa based on their topological roles. The topological role of each taxa was determined according to the scatter plot of within-module and among-module $\left(P_{i}\right)$ connectivities.

filtering relative to stochastic factors depends on the severity and duration of disturbance (Chase, 2007; Berga et al., 2012; Shade et al., 2012). Under these premises, it is expected that the determinism of the bacterial communities exhibited an elevated trend with increasing eutrophication levels (long-term and continuous disturbances), as indicated in our study (Fig. 4). Collectively, the magnitude of changes in the BCCs and the underlying deterministic processes depend on the eutrophication levels.

The sensitivity of bacterial communities to increasing eutrophication (Fig. 2) raises the question of whether sensitive taxa could serve as bio-indicators for eutrophication levels. As expected, we found that alterations in the relative abundance of 27 bacterial families were closely associated with eutrophication levels (Fig. 5), with the change pattern for a given bacterial family was concordant with its known ecological and biological functions. For example, bacterial families affiliated with Bacteroidetes (i.e., Cryomorphaceae, Flavobacteriaceae and Saprospiraceae) were the most abundant assemblages in the oligotrophic (low levels of inorganic nutrient, Table S1) Yushan Reserve (Fig. 5), which is contradict with their ability to better adapt to nutrient-enriched conditions (Dang and Lovell, 2016). However, a high relative abundance of Bacteroidetes lineages has been extensively detected in oceanic areas experiencing oligotrophic conditions (Blümel et al., 2007; Xiong et al., 2014). One possible explanation for this discrepancy is that Bacteroidetes are able to metabolize recalcitrant organic substrates (unaccessible to other lineages), thereby facilitating them an extra advantage in competitive exclusion (Fernández-Gómez et al., 2013). The relative abundance of some oligotrophic assemblages, such as Synechococcaceae and Oceanospirillaceae, were negatively correlated with eutrophication levels (Fig. 5). This pattern is consistent with the theory that they have evolved to be oligotrophic specialists (Andersson et al., 2010). In contrast, the relative abundances of copiotrophic families (i.e., Planctomycetaceae, Comamonadaceae and Nitrospiraceae) were enriched as eutrophication levels increased (Fig. 5). Planctomycetaceae and Comamonadaceae species are generally the predominant assemblages in soil (Newton et al., 2013; Wu et al., 2015), thus their greater relative abundances could be the result of inputs from terrestrial sources, delivered with freshwater run-off into Hangzhou Bay (Fig. S1). In addition, Nitrospiraceae species are the most common nitrite oxidizers known in nature (Daims, 2014). Considering a high level of DIN in Hangzhou Bay (Table S1), an increased relative abundance of Nitrospiraceae in turn might contribute essential roles in biogeochemical nitrogen cycling here. Indeed, high potential nitrification rates have been detected in water samples from this bay (Zhang et al., 2015). Even if correlation does not necessarily predict cause-effect relationship (bacterial changes might be consequence of other natural factors, rather than eutrophication alone), while the suspect compound (e.g., increasing eutrophication) that causes an observed effect can be deduced with strong stress-response associations (Boivin et al., 2002). Notably, the change patterns of these sensitive assemblages generally accord with their ecological strategies and are significantly associated with eutrophication levels (Fig. 5). For these reasons, the sensitive assemblages could be served as bioindicators for evaluating eutrophication levels, if bacterial responses consistently linked to the eutrophication levels over spatio-temporal scales.

Interspecies interaction drives the responses of a community to disturbance, which in turn determines the stability of an ecological community (Montoya et al., 2006; Shade et al., 2012). There is evidence, albeit limited, suggesting that increasing stress levels linearly enhance the strength (more densely connected between each other) of interactions among species (Thébault and Fontaine, 2010) and the importance of competition in driving extinction (Violle et al., 2010). It is expected, therefore, that the complexity of interspecies interaction is dependent on eutrophication levels. However, our findings showed that the complexity of interspecies interaction peaked under moderate eutrophications but declined under heavy eutrophication (Table 1). This pattern could be explained by moderate eutrophication which filters out the sensitive species from the endemic species pool, resulting in the co-occurrence of closely related bacterial lineages due to niche conservatism. In contrast, heavy eutrophication exerts random extinction and recolonization even among the core of regional taxa that were resistant to eutrophication. Consequently, the susceptibility to extinction might be decoupled from the species' traits, thereby causing less frequent interspecies interaction, less complex interactions, and fewer overlap of preferred environmental conditions or a lower degree of cooperative activities under heavy eutrophication (Table 1). Although a direct linkage between network complexity and community stability remains uncertain, it has been shown that the network complexity increases along successional gradients, resulting in greater stability of a food web (Neutel et al., 2007). Therefore, a microbial community could shift to an alternative stable state in response to moderate eutrophication. However, the buffer capacity of the bacterioplankton assembly (e.g., interspecies interaction) is limited, and could be disrupted altogether under heavy eutrophication. As such, heavy eutrophication could diminish the stability of a bacterial community, which, in turn, alters microbial-mediated function.

\section{Conclusion}

The magnitude of changes in BCCs and the importance of deterministic processes are closely associated with eutrophication levels. Compared with a random spatial distribution of bacterial communities in the undisturbed location, increasing eutrophication linearly attenuates the distance decay rates. In addition, the complexity of interspecies interaction is promoted by moderate eutrophication levels but is disrupted under heavy eutrophication. Notably, the relative abundances of the sensitive bacterial assemblages are significantly correlated with eutrophication levels, which could therefore serve as bio-indictors for evaluating eutrophication. This study represents a step forward in understanding the ecological consequences of increasing eutrophication and provides bio-indicators to evaluate coastal eutrophication. 


\section{Acknowledgements}

We appreciate the anonymous reviewers for their excellent comments. This work was supported by the Zhejiang Provincial Natural Science Foundation of China (LY15D060002), the Collaborative Innovation Center for Zhejiang Marine High-Efficiency and Healthy Aquaculture, and the K.C. Wong Magna Fund in Ningbo University.

\section{Appendix A. Supplementary data}

Supplementary data related to this article can be found at http:// dx.doi.org/10.1016/j.chemosphere.2017.03.034.

\section{References}

Ager, D., Evans, S., Li, H., Lilley, A.K., Van Der Gast, C.J., 2010. Anthropogenic disturbance affects the structure of bacterial communities. Environ. Microbiol. 12, 670-678.

Allison, S.D., Martiny, J.B., 2008. Resistance, resilience, and redundancy in microbial communities. Proc. Nat. Acad. Sci. U. S. A. 105, 11512-11519.

Andersson, A.F., Riemann, L., Bertilsson, S., 2010. Pyrosequencing reveals contrasting seasonal dynamics of taxa within Baltic Sea bacterioplankton communities. ISME J. 4, 171-181.

AQSIQ, 2007. The Specification for Marine Monitoring of China-Part 4: Seawater Analysis (GB 17378.4-2007). General administration of quality supervision, inspection and quarantine (AQSIQ) of the People's Republic of China (in Chinese).

Berga, M., Székely, A.J., Langenheder, S., 2012. Effects of disturbance intensity and frequency on bacterial community composition and function. PLoS One 7 , e36959.

Blümel, M., Süling, J., Imhoff, J.F., 2007. Depth-specific distribution of Bacteroidetes in the oligotrophic eastern mediterranean Sea. Aquat. Microb. Ecol. 46, 209-224.

Boivin, M.Y., Breure, A.M., Posthuma, L., Rutgers, M., 2002. Determination of field effects of contaminants-significance of pollution-induced community tolerance. Hum. Ecol. Risk Assess. 8, 1035-1055.

Borruso, L., Zerbe, S., Brusetti, L., 2015. Bacterial community structures as a diagnostic tool for watershed quality assessment. Res. Microbiol. 166, 38-44.

Bowen, J.L., Ward, B.B., Morrison, H.G., Hobbie, J.E., Valiela, I., Deegan, L.A., Sogin, M.L., 2011. Microbial community composition in sediments resists perturbation by nutrient enrichment. ISME J. 5, 1540-1548.

Chase, J.M., 2007. Drought mediates the importance of stochastic community assembly. Proc. Nat. Acad. Sci. U. S. A. 104, 17430-17434.

Daims, H., 2014. The Family Nitrospiraceae. The Prokaryotes. Springer, pp. 733-749.

Dang, H., Lovell, C.R., 2016. Microbial surface colonization and biofilm development in marine environments. Microbiol. Mol. Biol. Rev. 80, 91-138.

Deng, Y., Zhang, P., Qin, Y., Tu, Q., Yang, Y., He, Z., Schadt, C.W., Zhou, J., 2016. Network succession reveals the importance of competition in response to emulsified vegetable oil amendment for uranium bioremediation. Environ. Microbiol. 18, 205-218.

Deng, Y., Jiang, Y., Yang, Y., He, Z., Luo, F., Zhou, J., 2012. Molecular ecological network analyses. BMC Bioinforma. 13, 113.

Faust, K., Raes, J., 2012. Microbial interactions: from networks to models. Nat. Rev. Microbiol. 10, 538-550.

Fernández-Gómez, B., Richter, M., Schüler, M., Pinhassi, J., Acinas, S.G., González, J.M., Pedrós-Alió, C., 2013. Ecology of marine Bacteroidetes: a comparative genomics approach. ISME J. 7, 1026-1037.

Finlay, B.J., 2002. Global dispersal of free-living microbial eukaryote species. Science 296, 1061-1063.

Fodelianakis, S., Papageorgiou, N., Pitta, P., Kasapidis, P., Karakassis, I., Ladoukakis, E.D., 2014. The pattern of change in the abundances of specific bacterioplankton groups is consistent across different nutrient-enriched habitats in crete. Appl. Environ. Microbiol. 80, 3784-3792.

Hall, A.R., Miller, A.D., Leggett, H.C., Roxburgh, S.H., Buckling, A., Shea, K., 2012. Diversity-disturbance relationships: frequency and intensity interact. Biol. Lett. 8, 768-771.

Horner-Devine, M.C., Lage, M., Hughes, J.B., Bohannan, B.J., 2004. A taxa-area relationship for bacteria. Nature 432, 750-753.

Hou, W., Sun, S., Wang, M., Li, X., Zhang, N., Xin, X., Sun, L., Li, W., Jia, R., 2016. Assessing water quality of five typical reservoirs in lower reaches of Yellow River, China: using a water quality index method. Ecol. Indic. 61, 309-316.

Kembel, S.W., Cowan, P.D., Helmus, M.R., Cornwell, W.K., Morlon, H., Ackerly, D.D., Blomberg, S.P., Webb, C.O., 2010. Picante: R tools for integrating phylogenies and ecology. Bioinformatics 26, 1463-1464.

Kenkel, N.C., Orlóci, L., 1986. Applying metric and nonmetric multidimensional scaling to ecological studies: some new results. Ecology 67, 919-928.

Kolde, R., 2012. Pheatmap: pretty heatmaps. R. package version 61.

Labbate, M., Seymour, J.R., Lauro, F., Brown, M.V., 2016. Anthropogenic impacts on the microbial ecology and function of aquatic environments. Front. Microbiol. 7,
145.

Lepori, F., Malmqvist, B., 2009. Deterministic control on community assembly peaks at intermediate levels of disturbance. Oikos 118, 471-479.

Liang, Y., Zhang, X., Zhou, J., Li, G., 2015. Long-term oil contamination increases deterministic assembly processes in soil microbes. Ecol. Appl. 25, 1235-1243.

Martiny, J.B., Eisen, J.A., Penn, K., Allison, S.D., Horner-Devine, M.C., 2011. Drivers of bacterial $\beta$-diversity depend on spatial scale. Proc. Nat. Acad. Sci. U. S. A. 108, $7850-7854$.

Monier, A., Comte, J., Babin, M., Forest, A., Matsuoka, A., Lovejoy, C., 2015. Oceanographic structure drives the assembly processes of microbial eukaryotic communities. ISME J. 9, 990-1002.

Montoya, J.M., Pimm, S.L., Solé, R.V., 2006. Ecological networks and their fragility. Nature 442, 259-264.

Nekola, J., White, P., 1999. The distance decay of similarity in biogeography and ecology. J. Biogeogr. 26, 867-878.

Nemergut, D.R., Schmidt, S.K., Fukami, T., O'Neill, S.P., Bilinski, T.M., Stanish, L.F., Knelman, J.E., Darcy, J.L., Lynch, R.C., Wickey, P., 2013. Patterns and processes of microbial community assembly. Microbiol. Mol. Biol. Rev. 77, 342-356.

Neutel, A., Heesterbeek, J.A., van de Koppel, J., Hoenderboom, G., Vos, A. Kaldeway, C., Berendse, F., de Ruiter, P.C., 2007. Reconciling complexity with stability in naturally assembling food webs. Nature 449, 599-602.

Newton, R.J., Huse, S.M., Morrison, H.G., Peake, C.S., Sogin, M.L., McLellan, S.L., 2013. Shifts in the microbial community composition of gulf coast beaches following beach oiling. PLoS One 8, e74265.

Olesen, J.M., Bascompte, J., Dupont, Y.L., Jordano, P., 2006. The smallest of all worlds: pollination networks. J. Theor. Biol. 240, 270-276.

Primpas, I., Tsirtsis, G., Karydis, M., Kokkoris, G.D., 2010. Principal component analysis: development of a multivariate index for assessing eutrophication according to the European water framework directive. Ecol. Indic. 10, 178-183.

Pringault, O., Lafabrie, C., Avezac, M., Bancon-Montigny, C., Carre, C., Chalghaf, M. et al., 2016. Consequences of contaminant mixture on the dynamics and functional diversity of bacterioplankton in a southwestern Mediterranean coastal ecosystem. Chemosphere 144, 1060-1073.

R Development Core Team, 2014. R: a Language and Environment for Statistical Computing. The R foundation for statistical computing. Vienna, Austria. ISBN: 3900051-07-0. http://www.R-project.org/.

Shade, A., Peter, H., Allison, S.D., Baho, D.L., Berga, M., Bürgmann, H., Huber, D.H. Langenheder, S., Lennon, J.T., Martiny, J.B., 2012. Fundamentals of microbial community resistance and resilience. Front. Microbiol. 3, 417.

Shade, A., Read, J.S., Welkie, D.G., Kratz, T.K., Wu, C.H., McMahon, K.D., 2011. Resistance, resilience and recovery: aquatic bacterial dynamics after water column disturbance. Environ. Microbiol. 13, 2752-2767.

Shannon, P., Markiel, A., Ozier, O., Baliga, N.S., Wang, J.T., Ramage, D., Amin, N., Schwikowski, B., Ideker, T., 2003. Cytoscape: a software environment for integrated models of biomolecular interaction networks. Genome Res. 13, 2498-2504.

Smith, M.B., Rocha, A.M., Smillie, C.S., Olesen, S.W., Paradis, C., Wu, L., et al., 2015 Natural bacterial communities serve as quantitative geochemical biosensors. mBio 6 e00326-15.

SOA, 2015. Marine environment Bulletin of China 2014. State Oceanic Administration (SOA), People's Republic of China (in Chinese).

Stegen, J.C., Lin, X., Fredrickson, J.K., Chen, X., Kennedy, D.W., Murray, C.J. Rockhold, M.L., Konopka, A., 2013. Quantifying community assembly processes and identifying features that impose them. ISME J. 7, 2069-2079.

Stegen, J.C., Lin, X., Konopka, A.E., Fredrickson, J.K., 2012. Stochastic and deterministic assembly processes in subsurface microbial communities. ISME J. 6, 1653-1664.

Thébault, E., Fontaine, C., 2010. Stability of ecological communities and the architecture of mutualistic and trophic networks. Science 329, 853-856.

Van Der Gast, C.J., Ager, D., Lilley, A.K., 2008. Temporal scaling of bacterial taxa is influenced by both stochastic and deterministic ecological factors. Environ. Microbiol. 10, 1411-1418.

Van der Gucht, K., Cottenie, K., Muylaert, K., Vloemans, N., Cousin, S., Declerck, S., Jeppesen, E., Conde-Porcuna, J.M., Schwenk, K., Zwart, G., 2007 The power of species sorting: local factors drive bacterial community composition over a wide range of spatial scales. Proc. Nat. Acad. Sci. U. S. A. 104, 20404-20409.

Vanwonterghem, I., Jensen, P.D., Dennis, P.G., Hugenholtz, P., Rabaey, K., Tyson, G.W. 2014. Deterministic processes guide long-term synchronised population dynamics in replicate anaerobic digesters. ISME J. 8, 2015-2018.

Violle, C., Pu, Z., Jiang, L., 2010. Experimental demonstration of the importance of competition under disturbance. Proc. Nat. Acad. Sci. U. S. A. 107, 12925-12929.

Wang, K., Ye, X., Chen, H., Zhao, Q., Hu, C., He, J., Qian, Y., Xiong, J., Zhu, J., Zhang, D., 2015. Bacterial biogeography in the coastal waters of northern Zhejiang, East China Sea is highly controlled by spatially structured environmental gradients. Environ. Microbiol. 17, 3898-3913.

Webb, C.O., Ackerly, D.D., McPeek, M.A., Donoghue, M.J., 2002. Phylogenies and community ecology. Ann. Rev. Ecol. Syst. 33, 475-505.

Wu, J., Xiong, J., Hu, C., Shi, Y., Wang, K., Zhang, D., 2015. Temperature sensitivity of soil bacterial community along contrasting warming gradient. Appl. Soil Ecol. 94, 40-48.

Xiong, J., Xiong, S., Qian, P., Zhang, D., Liu, L., Fei, Y., 2016. Thermal discharge-created increasing temperatures alter the bacterioplankton composition and functional redundancy. AMB Express 6, 68.

Xiong, J., Chen, H., Hu, C., Ye, X., Kong, D., Zhang, D., 2015. Evidence of 
bacterioplankton community adaptation in response to long-term mariculture disturbance. Sci. Rep. 5, 15274.

Xiong, J., Ye, X., Wang, K., Chen, H., Hu, C., Zhu, J., Zhang, D., 2014. Biogeography of the sediment bacterial community responds to a nitrogen pollution gradient in the East China Sea. Appl. Environ. Microbiol. 80, 1919-1925.

Zhang, Q., Tang, F., Zhou, Y., Xu, J., Chen, H., Wang, M., Laanbroek, H.J., 2015. Shifts in the pelagic ammonia-oxidizing microbial communities along the eutrophic estuary of Yong River in Ningbo City, China. Front. Microbiol. 6, 1180.

Zhou, J., Deng, Y., Zhang, P., Xue, K., Liang, Y., Van Nostrand, J.D., Yang, Y., He, Z
Wu, L., Stahl, D.A., 2014. Stochasticity, succession, and environmental perturbations in a fluidic ecosystem. Proc. Nat. Acad. Sci. U. S. A. 111, 836-845.

Zhou, J., Liu, W., Deng, Y., Jiang, Y., Xue, K., He, Z., Yang, Y., Wang, A., 2013. Stochastic assembly leads to alternative communities with distinct functions in a bioreactor microbial community. mBio 4 e00584-12.

Zhu, J., Dai, W., Dong, C., Zhang, J., Xiong, J., 2016. Contrasting ecological processes and functional compositions between intestinal bacterial community in healthy and diseased shrimp. Microb. Ecol. 72, 975-985. 